Check for updates

London, UK

Cite this as: BMJ 2020;371:m4912 http://dx.doi.org/10.1136/bmj.m4912 Published: 17 December 2020

\title{
Covid-19: Evidence is lacking to support vitamin D's role in treatment and prevention
}

\section{Jacqui Wise}

There is "insufficient evidence" to support taking vitamin D solely to prevent or treat covid-19, say leading health bodies. ${ }^{1}$

Everyone should follow UK government advice, however, and take a vitamin D supplement throughout the autumn and winter to maintain bone and muscle health, according to the National Institute of Health Care Excellence (NICE), Public Health England (PHE), and the Scientific Advisory Committee on Nutrition (SACN).

The three bodies convened an expert panel to rapidly review the evidence from recent studies on vitamin $\mathrm{D}$ in relation to covid-19. They concluded that more research was needed, particularly high quality randomised controlled trials.

On 28 November PHE and NICE put out guidance that adults, young people, and children over four years should consider taking a daily supplement containing $10 \mu \mathrm{g}$ (400 international units) of vitamin D between October and early March because people do not make enough vitamin D from sunlight in these months. ${ }^{2}$ Some groups of people should consider taking a supplement throughout the year if they have little or no sunshine exposure-for example if they are housebound, usually wear clothes that cover up most of their skin, or if they have dark skin.

The panel agreed that low vitamin D status was associated with more severe outcomes from covid-19. It is not possible, however, to confirm causality because many of the risk factors for severe covid-19 outcomes are the same as the risk factors for low vitamin D levels. The serum concentration of vitamin D falls during a systemic inflammation which may occur during severe covid-19 illness and it is difficult to know if low vitamin D status causes poor outcomes or vice versa.

In June 2020, SACN conducted a rapid review which concluded that there was insufficient evidence to determine whether vitamin D supplementation could have a role in preventing respiratory tract infections. ${ }^{34}$ The evidence assessed included a widely cited systematic review and meta-analysis published in The BMJ in 2017 which reported some benefit. ${ }^{5}$

In the new rapid review SACN looked at an updated systematic review and meta-analysis which is currently on a preprint server and not yet peer reviewed. ${ }^{6}$ This reported a modest protective effect of vitamin D supplementation compared with placebo for respiratory tract infections. The panel, however, noted a number of limitations of the studies included in the meta-analysis.

When the panel considered the evidence for treating covid-19 using vitamin D supplements they concluded that a randomised controlled trial in secondary care from Spain was small and of very low quality. The trial used a very high dose of oral calcifediol, the circulating metabolite of vitamin $\mathrm{D}$, which is not commonly used in the UK. The panel also had concerns about differences in comorbidities between the two comparator groups and a lack of blinding.

Paul Chrisp, director of the Centre for Guidelines at NICE, said, "While there is insufficient evidence to recommend vitamin $\mathrm{D}$ for the prevention or treatment of covid-19 at this time, we encourage people to follow government advice on taking the supplement throughout the autumn and winter period. As research continues on the impact of vitamin $D$ on covid-19, we are continuing to monitor evidence as it is published and will review and update the guidance if necessary."

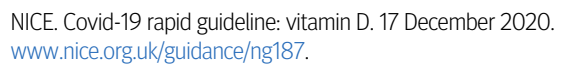

2 Statement from PHE and NICE on vitamin D supplementation during winter 28 November 2020. www.gov.uk/government/publications/vitamin-d supplementation-during-winter-phe-and-nice-statement/statement-fromphe-and-nice-on-vitamin-d-supplementation-during-winter.

3 Scientific Advisory Commission on Nutrition. Rapid review: vitamin D and acute respiratory tract infections. June 2020. https://app.box.com/s/g0ldpth1upfd7fw763ew3aqa3c0pyvky.

4 Martineau AR, Jolliffe DA, Hooper RL et al. Vitamin D supplementation to prevent acute respiratory tract infections: systematic review and meta-analysis of individual participant data. BMJ 2017;356:16583.

5 Torjesen I. Evidence does not support vitamin D for reducing respiratory infections, reviews conclude. BM/2020;369:m2629. doi: 10.1136/bmj.m2629 pmid: 32606036

6 Joliffe D, Carmargo CA, JrSluyter J, et al. Vitamin D supplementation to prevent acute respiratory infections: systematic review and meta-analysis of aggregate data from randomised controlled trials. MedRxiv 2020.07.14.20152728v1 [Preprint]. 2020. www.medrxiv.org/content/10.1101/2020.07.14.20152728v1doi: 10.1101/2020.07.14.20152728. 